ON THE NATURE OF VIBRATORY MOTIONS ${ }^{1}$ On the Nature of Sound

SOUND is the sensation peculiar to the ear. This sensation is caused by rapidly-succeeding to-and-fro motions of the air, which touches the outside surface of the drum-skin of the ear. These to-and-fro motions may be given to the air by a distant body, like a string of a violin. The string moves to and fro, that is, it vibrates. These vibrations of the string act on the bridge of the violin, which rests on the belly or sounding-board of the instrument. The surface of the sounding-board is thus set trembling, and these tremors, or vibrations, spread through the air in all directions around the instrument, somewhat in the manner that water-waves spread around the place where a stone has been dropped into a quiet pond. These tremors of the air, however, are not sound, but the cause of sound. Sound, as we have said, is a sensation; but, as the cause of this sensation is always vibration, we call those vibrations which give this sensation sonorous vibrations. Thus, if we examine attentively the vibrating string of the violin, we shall see that it looks like a shadowy spindle, showing that the string swings quickly to and fro; but, on closing the ears, the sensation of sound disappears, and there remains to us only the sight of the quick to-and-fro motion which, the moment before, caused the sound.

Behind the drum-skin of the ear is a jointed chain of three little bones. The one, $\mathrm{H}$ of Fig. I, attached to the

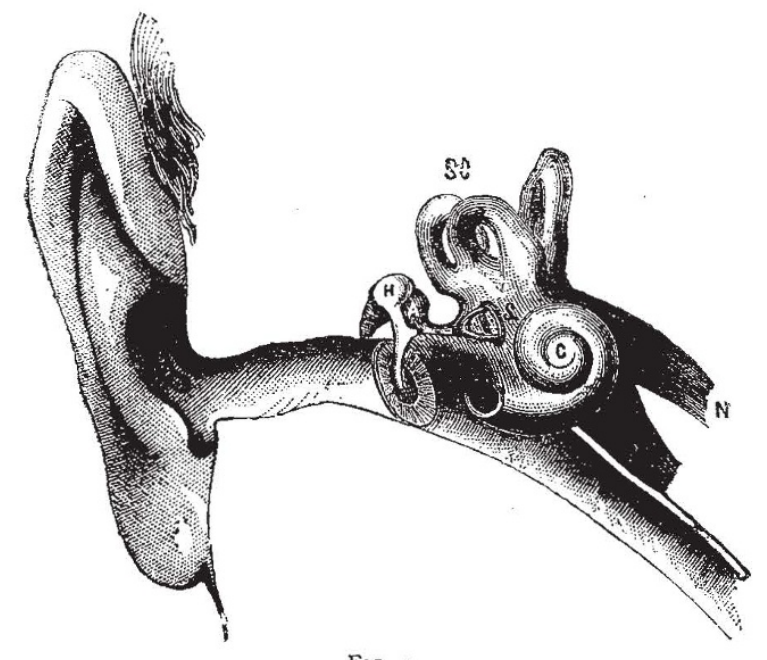

Fig. $x$.

drum-skin, is called the hammer; the next, A, is called the anvil; the third, $\mathrm{s}$, has the exact form of a stirrup, and is called the stirnu-bone. This last bone of the chain is attached to an oval membrane, which is a little larger than the foot of the stirrup. This oval membrane closes a hole opening into the cavity forming the inner ear; a cavity tunnelled out of the hardest bone of the head, and having a very complex form. The oval hole just spoken of opens into a globular portion of the cavity known as the vestibule, and from this lead three semicircular canals, $\mathrm{SC}$, and also a cavity, $\mathrm{C}$, of such a marked resemblance to a snail's shell that it is called cochlea, the Latin word for that object. The cavity of the inner ear is filled with a liquid, in which spread out the delicate fibres of the auditory nerve.

Let us consider how this wonderful little instrument acts when sonorous vibrations reach it. Imagine the violin-string vibrating 500 times in one second. The

"From a forthcoming work on "Sound: a Series of Simple, Enteriaining, and Inexpensive Experiments in the Phenomena of Scund, for the Use of Students of every Age." By Alfred Marshall Mayer, Professor of Physics in the Sievens Institute of Technology. Ccmmunicated by the author. sounding-board also makes 500 vibrations in a second. The air touching the violin is set trembling with 500 tremors a second, and these tremors speed with a velocity of 1,100 feet in a second in all directions through the surrounding air. They soon reach the drum-skin of the ear. The latter, being elastic, moves in and out with the air which touches it. Then this membrane, in its turn, pushes and pulls the little ear-bones 500 times in a second. The last bone, the little stirrup, finally receives the vibrations sent from the violin-string, and sends them into the fluid of the inner ear, where they shake the fibres of the auditory nerve 500 times in a second. These tremors of the nerve--how we know not--so affect the brain that we have the sensation which we call sound. The description we have just given is not that of a picture created by the imagination, but is an account of what really exists, and of what can actually be seen by the aid of the proper instruments.

A body may vibrate more or less frequently in a second; it may swing over a greater or less space; and it may have several minute tremors while it makes its main swing. These differences in vibrations make sounds higher or lower in pitch, loud or soft, simple or compound. It is easy to say all this, but really to understand it, one must make experiments and discover these facts for himself.

\section{On the Nature of Vibratory Motions}

The character of a sound depends on the nature of the vibrations which cause it, therefore our first experiments will be with vibrations which are so slow that we can study the nature of these peculiar motions. These experiments will be followed by others on vibrations of the same kind, only differing in this - that they are so rapid and frequent that they cause sounds. A correct knowledge of the nature of these motions lies at the foundation of a clear understanding of the nature of sound. We hope that the student will make these experiments with care, and keenly observe them.

Experiment 1.-At the toy-shops you can buy for a few pence a wooden ball having a piece of elastic rubber fastened to it. Take out the elastic and lay it aside, as we shall need it in another experiment. Get a piece of fine brass wire, about 2 feet (6I centimetres) long, and fasten it to the ball. The weight of the ball should pull the wire straight, and, if it does not, a finer wire must be used. Hold the end of the wire in the left hand, and with the right hand draw the ball to one side. Let it go, and it will swing backward and forward like the pendulum of a clock. This kind of movement we call a pendulous or transwerse vibration.

Experiment 2.-Cut out a narrow triangle of paper, 4 inches (10 centimetres) long, and paste it to the bottom of the ball. Twist the vire which supports the ball by turning the latter half round, and watch the paper pointer as it swings first one way and then the other. Here we have another kind of vibration, a motion caused by the twisting and untwisting of the wire. Such a motion is called a torsional vibration.

Experiment 3.- Take off the wire and the paper, and put the elastic on the ball. Hold the end of the elastic in one hand, and with the other pull the ball gently downward, then let it go. It vibrates up and down in the direction of the length of the elastic. Hence we call this kind of motion a longitudinal vibration.

These experiments show us the three kinds of vibrations, transverse, torsional, and longitudinal. They differ in direction, but all have the same manner of moving; for the different kinds of vibration, transverse, longitudinal, and torsional, go through motions with the same changes in velocity as take place in the swings of an ordinary pendulum. These vibrations all start from a position of momentary rest. The motion begins slowly, and gets faster and faster till the body gains the position it naturally has when it is at rest-at this point it has its 
greatest velocity. Passing this point, it goes slower and slower till it again comes momentarily to rest, and then begins its backward motion, and repeats again the same changes in velocity.

It is now necessary that the student should gain clear ideas of the nature of this pendulous motion. It is the cause of sound. It exists throughout all the air in which a sound may be perceived, and, by the changes in the number, extent of swing, and combinations of these pendular motions, all the changes of pitch, of intensity, and of quality of sound are produced. Therefore the knowledge which we now desire to give the reader lies at the very foundation of a correct understanding of the subject of this book.

An experiment is the key to this knowledge. It is the experiment with

\section{The Conical Pendulum}

An ordinary pendulum changes its speed during its swings right and left exactly as a ball appears to change its speed when this ball revolves with a uniform speed in a circle, and we look at it along a line of sight which is in the plane of the circle.

Experiment 4.- Let one take the ball and wire to the farther end of the room, and by a slight circular motion of the end of the wire he must cause the ball to revolve in a circle. Soon the ball gets into a uniform speed around the circle, and then it forms what is called a conical pendulum; a kind of pendulum sometimes used in clocks. Now stoop down till your eye is on a level with the ball. This you will know by the ball appearing

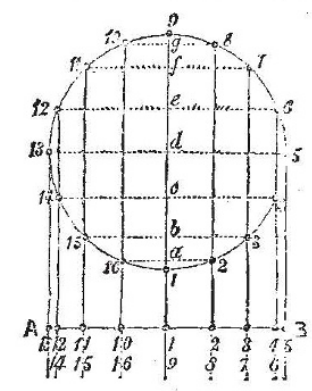

Fig. 2.

to move from side to side in a straight line. Study this motion carefully. It reproduces exactly the motion of an ordinary pendulum of the same length as that of the conical pendulum. From this it follows that the greatest speed reached during the swing of an ordinary pendulum just equals the uniform speed of the conical pendulum. That the apparent motion you are observing is really that of an ordinary pendulum you will soon prove for yourself to your entire satisfaction; and here let me say that one principle or fundamental fact seen in an experiment and patiently reflected on is worth a chapter of verbal descriptions of the same experiment.

Suppose that the ball goes round the circle of Fig. 2 in two seconds; then, as the circumference is divided into sixteen equal parts, the ball moves from I to 2 , or from 2 to 3 , or from 3 to 4 , and so on in one-eighth of a second. But to the observer who looks at this motion in the direction of the plane of the paper the ball appears to go from $I$ to 2 , from 2 to 3 , from 3 to 4 , \&C., on a line $A B$, while it really goes from 1 to 2 , from 2 to 3 , from 3 to 4 , \&c., in the circle. The ball when at $I$ is passing directly across the line of sight, and, therefore, appears with its greatest velocity; but when it is in the circle at 5 it is going away from the observer, and when at I 3 it is coming toward him, and, therefore, although the ball is really moving with its regular speed when at 5 and 13 , yet it appears when at these points momentarily at rest. From a comparison of the similarly numbered positions of the ball in the circle and on the line $A B$, it is evident that the ball appears to go from $A$ to $B$ and from $B$ back to $A$ in the time it takes to go from 13 round the whole circle to 13 again. That is the ball appears to vibrate from $A$ to $B$ in the time of one second, in which time it really has gone just half round the circle. A comparison of the unequal lengths I3 to I2, I2 to II, II to IO, \& $\mathrm{c}$, on the line A B, over which the ball goes in equal times, gives the student a clear idea of the varying velncity of a swinging pendulum.

Fig. 3 represents an upright frame of wood standing on a platform, and supporting a weight that hangs by a cord. A A is a flat board about 2 feet ( $6 \mathrm{r}$ centimetres) long and 14 inches ( 35.5 centimetres) wide. B B are two uprights so high that the distance from the under side of the cross-beam $\mathrm{C}$ to the platform A A is exactly $4 \mathrm{I} \frac{\mathrm{T}}{1} \mathrm{\sigma}$ inches (I metre and 45 millimetres). The crossbeam $\mathrm{C}$ is 18 inches $\left(45^{\circ} 7\right.$ centimetres) long. At $\mathrm{D}$ is a wooden post standing upright on the platform. Get a lead disk, or bob, $3 \frac{3}{16}$ inches (8 centimetres) in diameter; and $\frac{5}{8}$ inch ( 16 millimetres) thick. In the centre of this is a hole $\mathbf{I}$ inch ( 25 millimetres) in diameter. This disk may easily be cast in sand from a wooden pattern. At the tinner's we may have made a little tin cone $I_{1}^{3}$ inch ( 30

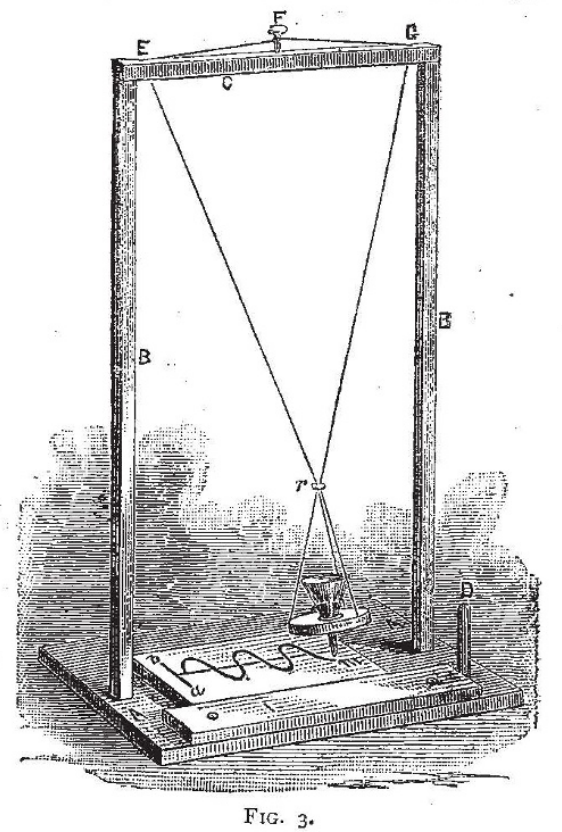

millimetres) wide at top, and $2 \frac{1}{4}$ inches ( 57 millimetres) deep, and drawn to a fine point. Carefully file off the point till a hole is made in the tip of the cone of about $\frac{1}{16}$ inch in diameter. Place the tin cone in the hole in the lead disk, and keep it in place by stuffing wax around it. A glass funnel, as shown in the figure, may be used instead of the tin cone. With an awl drill three small holes through the upper edge of the bob at equal distances from each other. To mount the pendulum, we need about 9 feet $\left(2 \mathrm{I}^{\circ} 5\right.$ centimetres) of fine strong cord, like trout-line. Take three more pieces of this cord, each ro inches $\left(25^{\circ} 4\right.$ centimetres) long, and draw one through each of the holes in the lead-bob and knot it there, and then draw them together and knot them evenly together above the bob, as shown in the figure. On the cross-bar, at the top of the frame, is a wooden peg shaped like the keys used in a violin. This is inserted in a hole in the bar-at $\mathrm{F}$ in the figure. Having done this, fasten one end of the piece of trout-line to the three cords of the bob, and pass the other end upward through the hole marked $\mathrm{E}$; then pass it through the hole in the key $\mathrm{F}$; turn the key round several times; then pass the cord through the 
hole at G, to the bob, and fasten it there to the cords. Then get a small bit of copper wire and bend it once round the two cords just above the knot, as at $r$ in the figure. This wire ring, and the upright post at the side of the platform, we do not need at present, but they will be used in future experiments with this pendulum.

Tack on the platform A.A a strip of wood $I$. This serves as a guide, along which we can slide the small board $m$, on which is tacked a piece of paper.

Experiment 5.-Fill the funnel with sand, and, while the pendulum is stationary, steadily slide the board under it. The running sand will be laid along LM, Fig. 4, in a straight line. If the board was slid under the sand during exactly two seconds of time, then the length of this line may stand for two seconds, and one-half of it may stand for one second, and so on. Thus, we see how time may be recorded in the length of a line.

Brush off the heaps of sand at the ends of the line, and

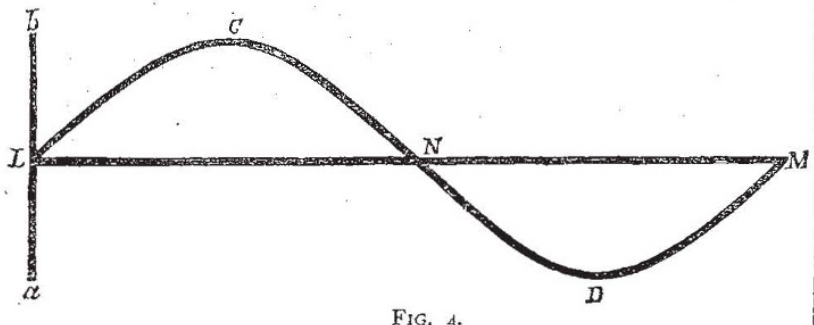

bring the left-hand end of the sand-line directly under the point of the funnel, when the latter is at rest. Draw the lead bob to one side, to a point which is at right angles to the length of the line, and let it go. It swings to and fro, and leaves a track of sand, $a b$, which is at right angles to the line L M, Fig. 4.

Suppose that the pendulum goes from $a$ to $b$, or from $b$ to $a$, in one second, and that, while the point of the funnel is just over $L$, we slide the board so that, in two seconds, the end $M$ of the line $L M$ comes under the point of the funnel. In this case the sand will be strewed by the pendulum to and fro, while the paper moves under it through the distance $x M$. The result is that the sand appears on the paper in a beautiful curve L C N D M. Half of this curve is on one side of $L M$, the other half on the opposite side of this line.

The experimenter may find it difficult to begin moving the paper at the very instant that the mouth of the funnel

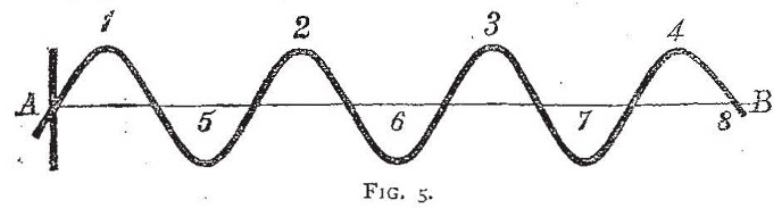

is over L; but, after several trials, he will succeed in doing this. Also, he need not keep the two sand-lines, $L M$ and $a b$, on paper during these trials; he may as well use their traces, made by drawing a sharply-pointed pencil through them on to the paper.

By having a longer board, or by sliding the board slowly under the pendulum, a trace with many waves in it may be formed, as in Fig. 5 .

As the sand-pendulum swung just like an ordinary pendulum when it made the wavy lines of Figs. 4 and 5 , it follows that these lines must be peculiar to the motion of a pendulum, and may serve to distinguish it. If so, this curve must have some sort of connection with the motion of the conical pendulum, described in Experiment 4 . This is so, and this connection will be found out by an attentive study of Fig. 6 .

In this figure we again see a wavy curve, under the same circular figure which we used in explaining how the motion of an ordinary pendulum may be obtained from the motion of a conical pendulum. This wavy curve is made directly from measures on the circular figure, and certainly bears a striking resemblance to the wavy trace made by the sand-pendulum in Experiment 5. You will soon see that to prove that these two curves are precisely the same is to prove that the apparent motion of the conical pendulum is exactly like the motion of the ordinary pendulum.

The wavy line of Fig. 6 is thus formed:-The dots on A $\mathrm{B}$, as already explained, show the apparent places of the ball on this line, when the ball really is at the points correspondingly numbered on the circumference of the circle. Without proof, we stated that this apparent motion on the line A B was exactly like the motion of a pendulum. This we must now prove. The line $L M$ is equal to the circumference of the circle stretched out. It is made thus :We take in a pair of dividers the distance I to 2 , or 2 to 3 , \&c., from the circle, and step this distance off sixteen times on the line $L M$; hence $L M$ equals the length of the circumference of the circle. In time this length stands for two seconds, for the ball in Experiment 4 took two seconds to go round the circle. This same length, you will also observe, was made in the same time as the sandline $L M$ was made in Experiment 5. In Fig. 6 the length $L M$, of two seconds, is divided into sixteen parts; hence each of them equals one-eighth of a second, just as the same lengths in the circle equal eighths of a second. Thus the line $\mathrm{LM}$ of Fig. 6, as far as a record of time is concerned, is exactly like the sand-line $L M$ of Experiment 5, and the line A B of Fig. 6, in which the ball appeared to move, is like the line $a b$ of Fig. 4, along which the sand-pendulum swung.

Now take the lengths from $I$ to $2, \mathrm{I}$ to $3, \mathrm{I}$ to $4, \mathrm{I}$ to 5 , and so on, from the line $A B$ of Fig. 6 , and place these lengths at right angles to the line $L M$ at the points $I, 2$, $3,4,5$, and so on; by doing so, we actually take the distances at which the ball appeared from I (its place of greatest velocity), and transfer them to $L M$; therefore, these distances correspond to the distances from L M, Fig. 4, to which the sand-pendulum had swung at the end of the times marked on L M of Fig. 6.

Join the ends of all these lines, $22^{\prime}, 33^{\prime}, 44^{\prime}$, \&c., by drawing a curve through them, and we have the wavy line of Fig. 6.

This curve evidently corresponds to the curve L C N D M of Fig. 4 made by the sand-pendulum; and it must be evident that, if this curre of Fig. 6 is exactly like the curve traced by the sand-pendulum in Experiment 7 , it follows that the apparent motion of the conical pendulum, as seen in the plane in which it revolves, is exactly like the real motion of an ordinary pendulum.

Experiment 6.--To test this, we make on a piece of paper one of the wavy curres exactly as we made the one in Fig. 6, and we tack this paper on the board $L M$ of 
the sand-pendulum, being careful that when the board is slid under the stationary pendulum the point of the funnel goes precisely over the centre line LM (Fig. 9) of the curve.

Now draw the point of the funnel aside to a distance from the line $\mathrm{L} M$ equal to one-half of A B, or, what is the same, from 5 to $5^{\prime}$ of Fig. 9. Pour sand in the funnel, and let the bob go. At the moment the point of the funnel is over $L$, slide the board along so that when the point of the funnel comes the third time to the line $L M$, it is at the end $M$ of this line. This you may not succeed in doing at first, but after several trials you will succeed, and then you will have an answer from the pendulum as to the kind of motion it has, for you will see the sand from the swinging pendulum strewed precisely over the curve you placed under it. Thus you have conclusively proved that the apparent motion of the conical pendulum, along the line $\mathrm{AB}$, is exactly like the swinging motion of an ordinary pendulum.

As it is difficult to start the board with a uniform motion at the very moment the pendulum is over the line $\mathrm{LM}$, it may be as well to tack a piece of paper on the board with no curve drawn on it, and then practise till you succeed in sliding the board under the pendulum, through the distance $\mathrm{L} M$, in exactly the time that it takes the pendulum to make two swings. Now, if you have been careful to have had the swing of your pendulum just equal to $A B$, or from 5 to $5^{\prime}$. on the drawing of the curve, you will have made a curve in sand which is precisely like the curve you have drawn; for, if you trace the sand-curve on the paper by carefully drawing through it the sharp point of a pencil, and then place this trace against a window-pane with the drawing of the curve, Fig. 6, directly over it, you will see that one curve lies directly over the other throughout all their lengths.

This curve, which we have made from the circle in Fig. 6, and have traced in sand by the pendulum, is called the curve of signs, or the sinusoid. It is so called because it is formed by stretching the circumference of a circle out into a line and then dividing this line, $L M$ of Fig. 6, into any number of equal parts. From the points of these divisions $\mathrm{I}, 2,3,4,5$, \&c., of $\mathrm{L} \mathrm{M}$, we erect perpendiculars $22^{\prime}, 33^{\prime}, 44^{\prime}, 55^{\prime}$, \&c., equal to the lines $a 2$, $b 3, c 4, d 5$, \&c., in the circle. These lines in the circle are called sines, so when we join the ends of these lines, erected to the straightened circumference by a curve, we form the curve of sines, or the sinusoid.

The sinusoid occurs often during the study of natural philosophy. We may meet with it again in our book on the nature of light, and it certainly will occur in our book on heat.

(To be continued.)

\section{NOTES}

$U_{P}$ to the present time the ignorance of those who did not know that the Archbishop of Canterbury was a degree-giving body was pardonable. It is so no longer. A serious alteration in the arrangements of these diplomas is now announced. Archbishop Tait, while he intends to dispense doctorates as before at his will and pleasure, has determined that his degree of M.A. is from December next to be a matter of examination. The stan. dard is to be that of "honour examinations in the Universities." There is to be due choice of subjects, among which, however, Greek and Latin are not to be compulsory, though English literature is. To qualify for examination, formal testimonials required for University matriculation, with the addition of a certificate from the Bishop of the diocese whence the candidates come, are required. As the Daily News puts it, "the Arch. bishop has evidently determined to make himself into a university with all the paraphernalia which the modern conception of such a body requires." Both the London Examining Board (commonly called the London University) and Owens College are to be congratulated on the publicity now given to this singular system of granting degrees. The London Examining Body is not a teaching body, neither is the Archbishop, but the Archbishop is a university, therefore the London University system is perfect, and all methods of education whatever may be disregarded so that a standard of instruction is reached. Owens College as a teaching centre which has won its way to general esteem and confidence, may now bide its time, for this last grotesque thing calling itself a university will either make the power of granting degrees, and degrees themselves ridiculous, or direct attention to the whole subject.

Although the Paris meeting of the Iron and Steel Institute has not called for any lengthy notice at our hands, there are passages in Dr. Siemens' admirable address to which we cannot too strongly draw attention, and which we are anxious to place on record in our columns. He remarked that "Whilst the English, to realise a novel proposition, make bold attempts, not always carefully matured beforehand, the French systematically study a question in all its aspects, and fortify their views by careful inquiry into the experience obtained elsewhere, before they commence operations which are then carried out with all the economical and other advantages resulting from such an exhaustive preliminary inquiry. If we seek a cause for the remarkable aptitude of adapting means to special ends, to which I have referred, we shall probably find it in the advantages France and other continental countries have enjoyed for at least a generation of a more extended technical education than we could boast of, and of the personal influence which has been exercised by a line of scientific writers and experimentalists, of whom I shall only mention here such honoured names as those of Réaumur, Ebelmen, Régnault, Pouillet, Péclet, Thomas, and Le Châtelier, as belonging to the past, and of Deville, Griiner, Lan, Laurens, Jordan, Frémy, and Dumas, who are fortunately still among us. It is chiefly to such men as these that France owes her admirable system of education, which enables her to place her metallurgical establishments under the guidance of men who are scientifically qualified for the discharge of their respective duties, and for the attainment of practical results which may well excite our admiration." The organisation of the Ecole Centrale, the creation of $\mathbf{M}$. Dumas, recommends itself, as it may well do, to Dr. Siemens, and he points out that the only establishment in Great Britain comparable with the École Centrale as regards metallurgy is our School of Mines, which, "if it were installed in a capacious building, and had other branches of knowledge added to its curriculum, might easily, under the guidance of such men as Percy, Smyth, Frankland, and Huxley, be developed into an institu. tion which would give rise to beneficial results difficult to overestimate." Had Dr. Siemens been speaking in England he would doubtless have added that this was the distinct recommendation made by the Duke of Devonshire's Commission after. a long inquiry. The Government has not yet acted upon this recommendation, and the result is that students of the School of Mines have to get their mathematics when and how they can; they form no part of the curriculum. Many may think that such schools in France are too heavily weighted with mathematics, but to omit the subject altogether is to court Scylla with a vengeance. Why should not each student of the School of Mines receive, as at the École Centrale, a three years' course of general scientific education, including the higher branches of mathematics, as well as physical science, pure and applied chemistry, geology, mechanics, metallurgy, and mineralogy.

MR. H. Forbes, F.L.S., is about to leave this country to investigate the fauna and fiora of Celebes, Borneo, and adjacent islands. He proposes to devote five or six years to the work.

Vesuvius is now giving some very definite signs of an eruption. 